\title{
Internal mode dynamics in driven nonlinear Klein-Gordon systems
}

\author{
N.R. Quintero ${ }^{1}$, A. Sánchez ${ }^{1, a}$, and F.G. Mertens ${ }^{2}$ \\ 1 Grupo Interdisciplinar de Sistemas Complicados (GISC), Departamento de Matemáticas, Universidad Carlos III de Madrid, \\ Edificio Sabatini, Avenida de la Universidad 30, 28911 Leganés, Madrid, Spain \\ 2 Physikalisches Institut, Universität Bayreuth, 95440 Bayreuth, Germany
}

Received 6 June 2000

\begin{abstract}
We study analytically and numerically the action of a constant force on the propagation of kinks in the $\phi^{4}$ and sine-Gordon systems, with and without dissipation. We specifically investigate the relation of the external force with the oscillations of the kink width due to excitation of its internal mode or quasimode. We demonstrate that both dc force and dissipation, either jointly or separately, damp the oscillations of the kink width. We further prove that, in contrast to earlier predictions, those oscillations can only arise if we use a distorted kink as initial condition for the evolution. Finally, we show that for the $\phi^{4}$ system the oscillations of the kink width come from the excitation of its internal mode, whereas in the $\mathrm{sG}$ equation they originate in the excitation of the lowest radiational modes and an internal mode induced by the discreteness of the numerical simulations.
\end{abstract}

PACS. 05.45.-a Nonlinear dynamics and nonlinear dynamical systems - 63.20.Ry Anharmonic lattice modes $-74.50 .+\mathrm{r}$ Proximity effects, weak links, tunneling phenomena, and Josephson effects - 85.25.Cp Josephson devices

\section{Introduction}

More than thirty years after the concept of soliton entered physics, their dynamics, as well as that of other coherent structures emerging from nonlinearity, is still ridden with surprising new phenomena [1]. Even though some facts have been well established - for instance, the particlelike behavior of many nonlinear coherent structures in the presence of a variety of perturbations $[2,3]$ - other features are much worse understood. Prominent among the latter is the interplay of the translational motion of a coherent structure with its internal degrees of freedom or modes: First found in several pioneering works [4] dealing with resonant exchange of energy between solitary waves through their internal mode, the importance of this possible coupling has not been studied in depth until the last few years. Indeed, only recently it has been realized that the coupling between translation and internal modes becomes the governing physical factor in many contexts, such as interactions with inhomogeneities [5], with thermal noise [6] or with external drivings [7], to name a few. Furthermore, it has also been shown that even in systems where the relevant coherent structure does not have internal modes, these can arise due to the presence of very small perturbations of different types $[8,9]$; hence their importance and generality.

A specific class of models where internal modes are relevant is the nonlinear Klein-Gordon equation, the kink

\footnotetext{
a e-mail: anxo@math.uc3m.es
}

solitary waves of the sine-Gordon ( $\mathrm{sG}$ ) and $\phi^{4}$ equation being most interesting because of their very many physical applications (see [1] and references therein). In the early eighties, Rice introduced [10] a variational approach to deal with internal modes of nonlinear Klein-Gordon kinks. Interestingly, whereas Rice's approach yielded very accurate results for the internal mode of $\phi^{4}$ kinks, it also predicted an internal mode for sG kinks. The latter result was quite striking, since linear stability analysis of sG kinks does not show any internal, localized mode, and, in addition, the putative internal mode of Rice's approach turned out to be within the phonon (linear radiation modes) band. Nevertheless, a few years later Boesch and Willis reported [11] numerical observations of long-lived oscillations of the width of sG kinks (which they termed "quasimode") which they related to Rice's mode. Moreover, Majerníková, Gaididei and Braun theoretically predicted [12], by a collective coordinate (CC) approach, that the internal mode of $\mathrm{sG}$ and $\phi^{4}$ kinks should be observed in the dynamics of kinks driven by a constant, external force; however, they did not carry out numerical simulations to check their results. After a decade of conjectures, we recently showed [13] that sG kinks do not have any internal mode of the kind predicted by Rice and that the observations in [11] were in fact due to the lowest phonon mode. It is then natural to ask whether or not the theoretical results of [12] compare well with the dynamics of kinks in simulations of the full partial differential equations. In this paper, we set out to clarify this point beginning by 
looking again at the CC calculations in [12]. In Section 2 we obtain the equations for the evolution of the position and width of $\mathrm{sG}$ and $\phi^{4}$ kinks and analyze them for arbitrary initial conditions, noting that the results in [12] are limited to a specific choice of the initial values and to the dissipation-free case. In Section 3 we summarize the details of our numerical simulation procedure, as well as the computational definitions of the kink width. Section 4 is devoted to the comparison of the analytical and numerical results, both with and without dissipation. Finally, our conclusions are presented in Section 5.

\section{Collective coordinate approach}

As announced above, we study kink propagation in the $\phi^{4}$ and sine-Gordon models when perturbed by a constant force $\epsilon$, and damping with coefficient $\beta$,

$$
\phi_{t t}-\phi_{x x}=-\frac{\mathrm{d} U}{\mathrm{~d} \phi}-\beta \phi_{t}-\epsilon
$$

where $U(\phi)=1-\cos (\phi)$ for $\mathrm{sG}$ or $U(\phi)=\left(\phi^{2}-1\right)^{2} / 4$ for $\phi^{4}$. As in previous works $[10,12]$, to address this problem we begin by assuming that the solution of this equation has the form

$$
\phi(x, t)=\phi_{0}\left(\frac{x-X(t)}{l(t)}\right)
$$

where $\phi_{0}$ is the static kink solution of the unperturbed $\phi^{4}$ or sG equations $(\epsilon=0$ and $\beta=0$ in Eq. (1)), and $X(t)$ and $l(t)$ represent the position of the center of the kink and its width, respectively. In order to find the equations governing the evolution of the two CC $X(t)$ and $l(t)$, we can either use a projection method proposed in [14] (see also [7]), or the variations of the energy and the momentum of the system [15]. Introducing the momentum of the perturbed system,

$$
P(t)=M_{0} l_{0} \frac{\dot{X}(t)}{l(t)},
$$

where $M_{0}$ is the rest energy and $l_{0}$ is the width of the static kink, both procedures lead straightforwardly to

$$
\frac{\mathrm{d} P}{\mathrm{~d} t}=-\beta P+q \epsilon,
$$

$q$ being the kink topological charge, and

$$
\alpha\left[\dot{l}^{2}-2 l \ddot{l}-2 \beta l i\right]=\frac{l^{2}}{l_{0}^{2}}\left[1+\frac{P^{2}}{M_{0}^{2}}\right]-1 .
$$

The parameters in these equations are, $q=2, \alpha=$ $\left(\pi^{2}-6\right) / 12, M_{0}=4 /\left(3 l_{0}\right)$, and $l_{0}=\sqrt{2}$ for $\phi^{4}$ kinks, and $q=2 \pi, \alpha=\pi^{2} / 12, M_{0}=8$, and $l_{0}=1$ for $\mathrm{sG}$ kinks. Integrating equation (4) when $\beta=0$ we find

$$
P(t)=P(0)+q \epsilon t, \quad P(0)=\frac{M_{0} \dot{X}(0) l_{0}}{l_{s}},
$$

which is linear in $t$, whereas when $\beta \neq 0$,

$$
P(t)=P(0) \exp (-\beta t)-\frac{q \epsilon}{\beta}[\exp (-\beta t)-1],
$$

i.e., $P(t)$ decreases exponentially in time up to the value $q \epsilon / \beta$. We thus have the well known result [2] that kinks are accelerated by the constant force, $\epsilon$, and decelerated by the dissipation, $\beta$; when both are present, kinks achieve an asymptotic, steady velocity arising from the balance of the two forces.

We now move to the equation for the width of the kink, $l(t)$, given by equation (5). To solve it, we follow [12] and change variables according to $l(t)=g^{2}(t)$, which gives

$$
\ddot{g}+\beta \dot{g}+\left(\frac{\Omega_{\mathrm{R}}}{2}\right)^{2}\left[1+\left(P(t) / M_{0}\right)^{2}\right] g=\frac{1}{4 \alpha g^{3}},
$$

with the Rice frequency $[10] \Omega_{\mathrm{R}}=1 /\left(\sqrt{\alpha} l_{0}\right)$. We have not been able to solve the dissipative case, $\beta \neq 0$, and therefore we will numerically integrate equation (8) in order to compare with the simulation results in Section 4. However, the dissipation-free case can be solved. We treat this case in detail in the following subsection.

\subsection{Solution of the dissipation-free problem}

When $\beta=0$, we have an Ermakov-type equation $[16,17]$,

$$
\ddot{g}+\left(\bar{\Omega}^{2}+\bar{\beta}^{2}\left[t+\frac{P(0)}{q \epsilon}\right]^{2}\right) g=\frac{1}{4 \alpha g^{3}},
$$

where $\bar{\Omega}=\Omega_{\mathrm{R}} / 2$ and $\bar{\beta}=q \epsilon \bar{\Omega} / M_{0}$, with initial conditions

$$
g(0)=\sqrt{l_{s}}, \quad \dot{g}(0)=\frac{\dot{l}(0)}{2 \sqrt{l_{s}}},
$$

where $l_{s}=l_{0} \sqrt{1-\dot{X}(0)^{2}}$ is the initial Lorentz-contracted kink width. The solution of this equation is given by [17]

$$
g(t)=\sqrt{v_{1}^{2}(t)+\frac{1}{4 \alpha W^{2}} v_{2}^{2}(t)}
$$

where the nonzero constant $W=v_{1}(t) \dot{v}_{2}(t)-\dot{v}_{1}(t) v_{2}(t)$ is the Wronskian and the functions $v_{m}(t)$ fulfill

$$
\begin{gathered}
\ddot{v}_{m}+\left(\bar{\Omega}^{2}+\bar{\beta}^{2}\left[t+\frac{P(0)}{q \epsilon}\right]^{2}\right) v_{m}=0, \quad m=1,2, \\
v_{1}(0)=\sqrt{l_{s}}, \quad \dot{v}_{1}(0)=\frac{i(0)}{2 \sqrt{l_{s}}} \\
v_{2}(0)=0, \quad \dot{v}_{2}(0)=\text { const. } \neq 0 .
\end{gathered}
$$

With a new change of variables $\tau$ and $Y_{m}$ given by [12]

$$
\tau=\frac{1}{2}\left[t-\frac{P(0)}{q \epsilon}\right]^{2}, \quad Y_{m}(\tau)=\tau^{1 / 4} v_{m}(\tau),
$$


Equation (12) is nothing but the Whittaker equation [18],

$$
Y_{m}^{\prime \prime}(\tau)+\left[\bar{\beta}^{2}+\frac{\bar{\Omega}^{2}}{2 \tau}+\frac{3}{16 \tau^{2}}\right] Y_{m}(\tau)=0 .
$$

The independent solutions of (14) are the Whittaker functions [19] $Y_{m}(\tau)=M_{r, \pm 1 / 4}(2 \mathrm{i} \bar{\beta} \tau)$, where $r=-\mathrm{i} \bar{\Omega}^{2} /(4 \bar{\beta})$. Therefore, the solution of equation (12) can be written as the linear superposition of those functions,

$$
\begin{aligned}
v_{m}(t)=\frac{1}{\tau^{1 / 4}} & {\left[A_{m} M_{r, 1 / 4}(2 \mathrm{i} \bar{\beta} \tau)+\right.} \\
& \left.+B_{m} M_{r,-1 / 4}(2 \mathrm{i} \bar{\beta} \tau)\right],
\end{aligned}
$$

where the constants $A_{m}$ and $B_{m}$,

$$
\begin{aligned}
A_{1}= & \frac{\mathrm{i} \tau_{0}^{1 / 4}}{\bar{\beta}}\left[\sqrt{l_{s}} M_{r,-1 / 4}^{\prime}\left(2 \mathrm{i} \bar{\beta} \tau_{0}\right)\right. \\
& \left.-\left(\frac{\sqrt{l_{s}}}{4 \sqrt{\tau_{0}}}-\frac{q \epsilon}{P(0)} \frac{i(0)}{2 \sqrt{l_{s}}}\right) M_{r,-1 / 4}\left(2 \mathrm{i} \bar{\beta} \tau_{0}\right)\right], \\
B_{1}= & \frac{\mathrm{i} \tau_{0}^{1 / 4}}{\bar{\beta}}\left[-\sqrt{l_{s}} M_{r, 1 / 4}^{\prime}\left(2 \mathrm{i} \bar{\beta} \tau_{0}\right)\right. \\
& \left.+\left(\frac{\sqrt{l_{s}}}{4 \sqrt{\tau_{0}}}-\frac{q \epsilon}{P(0)} \frac{i(0)}{2 \sqrt{l_{s}}}\right) M_{r, 1 / 4}\left(2 \mathrm{i} \bar{\beta} \tau_{0}\right)\right], \\
A_{2}= & \frac{\mathrm{i} \tau_{0}^{1 / 4}}{\bar{\beta}}\left[\frac{q \epsilon}{P(0)} \dot{v}_{2}(0) M_{r,-1 / 4}\left(2 \mathrm{i} \bar{\beta} \tau_{0}\right)\right] \\
B_{2}= & \frac{\mathrm{i} \tau_{0}^{1 / 4}}{\bar{\beta}}\left[-\frac{q \epsilon}{P(0)} \dot{v}_{2}(0) M_{r, 1 / 4}\left(2 \mathrm{i} \bar{\beta} \tau_{0}\right)\right] \\
\tau_{0}= & \frac{1}{2}\left[\frac{P(0)}{q \epsilon}\right]^{2}
\end{aligned}
$$

have been calculated from the initial conditions. This result generalizes that in [12], which was obtained for a restricted set of initial conditions, namely $l(0)$ was fixed to $l_{0}$ after arbitrarily setting $A_{2}=B_{1}=0$ (see [12]). Therefore, the result (15) with constants given by equation (16) is obtained here for the first time with full generality.

Taking into account the relations (15) and (16), we can finally write the solution for the width of the kink as

$$
l(t)=v_{1}^{2}(t)+\frac{1}{4 \alpha W^{2}} v_{2}^{2}(t), \quad W=\sqrt{l_{s}} \dot{v}_{2}(0) .
$$

In the particular case of zero initial velocity, a simpler expression can be obtained: equations (15) and (16) reduce then to

$$
\begin{aligned}
v_{m}(t)= & \frac{1}{t^{1 / 2}}\left[A_{m} M_{r, 1 / 4}\left(\mathrm{i} \bar{\beta} t^{2}\right)+\right. \\
& \left.\quad+B_{m} M_{r,-1 / 4}\left(\mathrm{i} \bar{\beta} t^{2}\right)\right], \\
A_{1}= & \frac{i(0)}{2 \sqrt{l_{s}}(\mathrm{i} \bar{\beta})^{3 / 4}}, \\
B_{1}= & \frac{\sqrt{l_{s}}}{(\mathrm{i} \bar{\beta})^{1 / 4}}, \\
A_{2}= & \frac{\dot{v}_{2}(0)}{(\mathrm{i} \bar{\beta})^{3 / 4}}, \\
B_{2}= & 0 .
\end{aligned}
$$

We can now use equation (6) for the momentum and equation (17) (Eq. (18) when the initial velocity is zero) along with the relationship $\dot{X}(t)=P(t) l(t) / M_{0} l_{0}$ to find an analytical expression for the kink velocity as predicted by the CC approach. However, from that expression we have not been able to find analytically $X(t)$, and therefore in Section 4 we compare the evolution of the kink width and velocity (and not the evolution of the kink position) with the numerical simulations of the full partial differential equations.

\section{Numerical simulation details}

In order to check our CC results, we will integrate numerically equation (1), corresponding to the perturbed $\phi^{4}$ and sG models, using the Strauss-Vázquez [20] method. With this energy-conserving scheme we can accurately compute the center and the velocity of the kink through the energy and the momentum of the system [21]. To obtain the numerical values for the kink center we have also used as an additional test the method proposed in [22] for the overdamped $\mathrm{sG}$ system, in which the kink center is defined as the point $X_{\text {num }}$ at which $\phi\left(X_{\text {num }}, t\right)=\pi$ for the $\mathrm{sG}$ $\left(\phi\left(X_{\text {num }}, t\right)=0\right.$ for $\left.\phi^{4}\right)$. In order to compute $X_{\text {num }}$, we first find the points $x(n)$ and $x(n+1)$ of the computational grid such that $\phi^{n} \leq \pi$ and $\phi^{n+1} \geq \pi$ for sG $\left(\phi^{n} \leq 0\right.$ and $\phi^{n+1} \geq 0$ for $\phi^{4}$ ), and we then interpolate linearly between $n$ and $n+1$, thus finding the numerical value of the kink center. Once we have obtained $X_{\text {num }}(t)$, we can compute the kink width, $l_{\text {num }}(t)$, finding the value of $l(t)$ that minimizes the expression

$$
\sum_{n=1}^{N}\left|\phi^{n}(t)-\phi_{\text {theo }}^{n}(t)\right|^{2},
$$

where

$$
\phi_{\text {theo }}^{n}(t)=\tanh \left[\frac{x(n)-X_{\text {num }}(t)}{l(t)}\right]
$$

for $\phi^{4}$, and

$$
\phi_{\text {theo }}^{n}(t)=4 \arctan \left[\exp \left(\frac{x(n)-X_{\text {num }}(t)}{l(t)}\right)\right]
$$


for sG. In the above equations, $\phi^{n}(t)$ stands for the numerical solution of equation (1), whereas $\phi_{\mathrm{theo}}^{n}(t)$ represents the discrete version of the analytical static kink solution, centered at $X_{\text {num }}(t)$ with width $l(t)$. In order to minimize equation (19) we let $l(t)$ vary between $l_{\min }$ and $l_{\max }$, with a precision of 0.001 . We stress that, to find $l(t)$, equation (19) is most appropriate to compare with the CC results of the last section. Finally, we have used $X_{\text {num }}(t)$ to calculate the velocity of the kink center

$$
\dot{X}_{\text {num }}(t)=\frac{X_{\text {num }}(t+\Delta t)-X_{\text {num }}(t)}{\Delta t} .
$$

With respect to initial conditions, we have started from kinks initially centered at $X(0)$, with width $l(0)$ and initial velocity $\dot{X}(0)$, i.e.,

$$
\begin{aligned}
\phi^{n}(0)= & \tanh \left[\frac{x(n)-X(0)}{l(0)}\right], \\
\phi_{t}^{n}(0)= & \frac{1}{\cosh ^{2}\left[\frac{x(n)-X(0)}{l(0)}\right]} \\
& \times\left[-\frac{u(0)}{l(0)}-\frac{x(n)-X(0)}{l^{2}(0)} i(0)\right],
\end{aligned}
$$

for $\phi^{4}$, and

$$
\begin{aligned}
\phi^{n}(0)= & 4 \arctan \left(\exp \left[\frac{x(n)-X(0)}{l(0)}\right]\right), \\
\phi_{t}^{n}(0)= & \frac{2}{\cosh \left[\frac{x(n)-X(0)}{l(0)}\right]} \\
& \times\left[-\frac{u(0)}{l(0)}-\frac{x(n)-X(0)}{l^{2}(0)} i(0)\right],
\end{aligned}
$$

for $\mathrm{sG}$, respectively. It is important to notice that in equations $(23,24)$ we can impose $l(0) \neq l_{s}$ and $l(0) \neq 0$, giving rise to a distorted initial kink; we will see below that this is indeed the most relevant situation for the appearance of kink width oscillations.

The parameters of the discretizations of equation (1) are $\Delta x=0.1,0.05, \Delta t=0.01,0.005$ and $2 L=100,200$. Furthermore, we have varied $\epsilon$ between 0.001 and 0.02 . For these values of $\epsilon$, we have found that $l(0)$ and $l(0)$ should not differ too much from $l_{s}$ and 0 : Indeed, for $l(0)=l_{s}+1$ or $i(0)=1$ we observe radiation in the system, and even breathers or kink-antikink pairs can spontaneously appear. Therefore, we stick to smaller deviations which do not modify the single kink propagation scenario.

\section{Results}

We begin by comparing the predictions of equations (1518) for the evolution of the kink width under a constant force and without dissipation. In this context, it is important to recall that the kink width, $l(t)$, depends not only on the initial kink velocity, $\dot{X}(0)$, and on the amplitude of the external force, $\epsilon$, but also on the initial conditions $l(0)$ and $i(0)$, which was not considered in [12] (they do not have numerical results to compare with in any case), hence the importance of exploring all possible choices for $l(0)$ and $\dot{l}(0)$. We present our results in the following manner: In all cases we have taken $\dot{X}(0)=0$ and there are two plots for the same initial conditions in each figure, one corresponding to $\phi^{4}$ (upper panel) and the other to sG (lower panel). In all figures, solid lines represent analytical results obtained from the $\mathrm{CC}$ theory and dotted lines correspond to numerical simulations.

Figure 1 presents results for driven kink propagation with initial conditions given by an undistorted kink shape. At the CC level, this means that $l(0)=l_{s}$ and $l(0)=0$. As may be seen, the agreement between the theoretical approach and the numerical results is excellent, which supports the appropriateness of our definitions for the numerical kink center and width. In the plots, we see that in both systems the kink width (left figures) does not oscillate, as predicted by the CC approach. In this figure we observe that $l(t)$ decreases, while, at the same time, the velocity of the kink center increases due to the action of the external force (right figures). Note that the numerically computed velocities are overimposed to the solid lines of the theoretical prediction. The small oscillations and irregularities observed in Figure 1 on the left are a consequence of the numerical discretization (notice the smallness of the vertical scale, of the order of the spatial integration step); we have verified that when we decrease the values of $\Delta x$ and $\Delta t$, the amplitude of those oscillations decreases as well. Finally, we observe that for the same final time of integration, the velocity of the center for the $\phi^{4}$ kink is almost equal to 0.4 , whereas the corresponding velocity for the sG kink is close to 0.15 . These velocities, computed numerically, agree very well with those predicted by the CC method.

If we now start our simulations from a distorted kink, choosing, i.e., $l(0) \neq l_{s}$ or/and $i(0) \neq 0$, the results change qualitatively, as shown in Figures 2 and 3, where it is clear that in this case $l(t)$ does oscillate. Results are similar for other values for $l(0)$ and $\dot{l}(0)$ with the above caveat that they should not differ too much from $l_{s}$ and 0 , respectively. For the $\phi^{4}$ kink, the amplitude of $l_{\text {num }}(t)$ is somewhat smaller than the prediction of the CC approach, and it slowly decreases, although it seems to be an excitation with a large lifetime. All in all, we can say that there is a reasonable agreement between theory and simulations. On the contrary, in the sG case, the oscillations of $l_{\text {num }}(t)$ are rapidly damped out, opposite to what we expected from the analytical method. For better comparison, we have used a discrete Fourier transform (DFT) to obtain the oscillation frequencies of $l(t)$ : the so computed frequency of the behavior predicted by the $\mathrm{CC}$ approach (solid lines in Figs. 2 and 3) yields $\omega_{\mathrm{CC}}=1.2568$ for the $\phi^{4}$ case, to be compared to the Rice value $\Omega_{\mathrm{R}}=1.2452$. It is then natural to associate the oscillations of the $\phi^{4}$ kink width to the frequency of the internal mode, $\Omega_{\mathrm{i}}=1.2247$, and indeed, the result obtained from the numerical simulation 

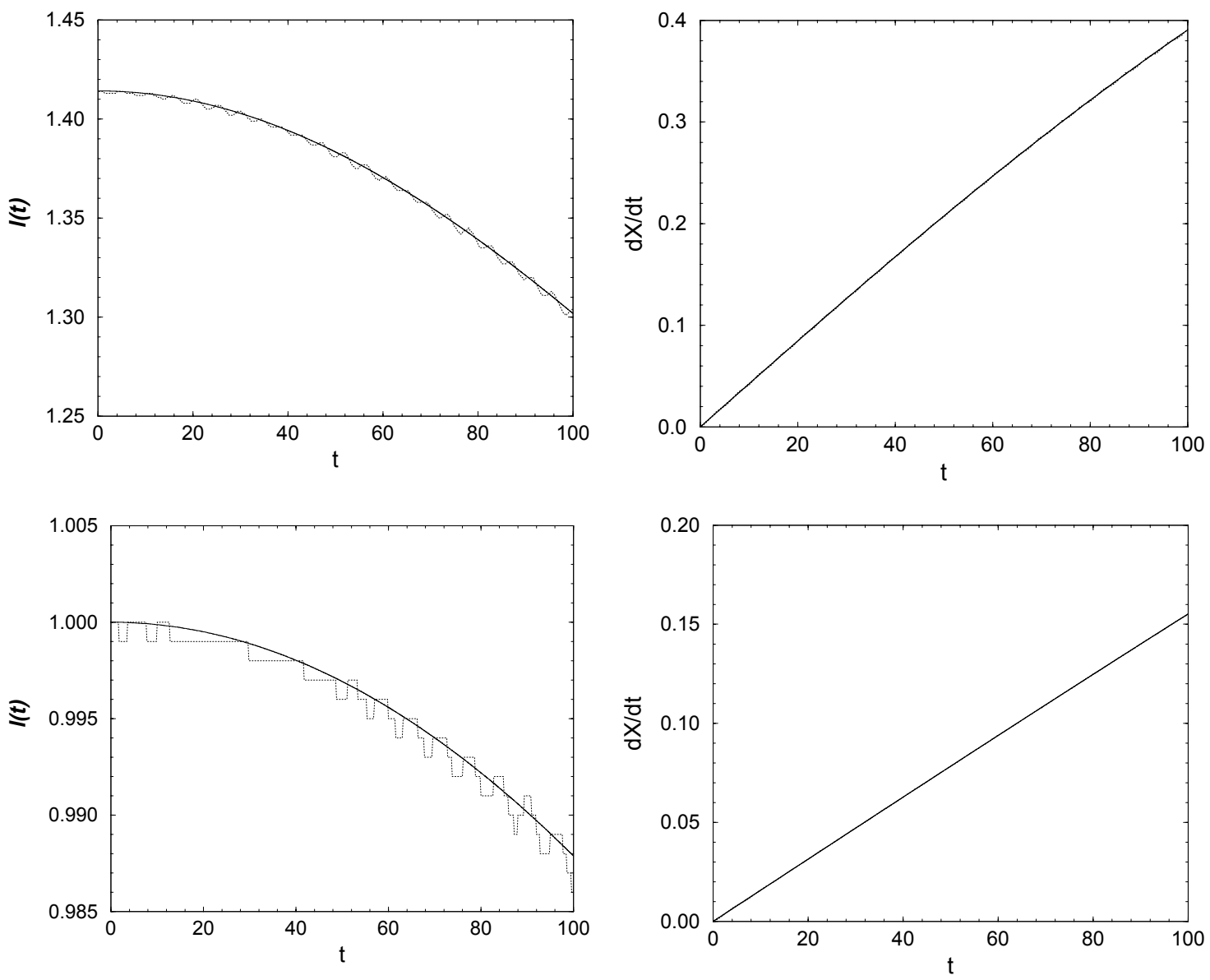

Fig. 1. Left: Evolution of the kink width, $l(t)$, theoretically obtained from equations $(17,18)$ (solid lines), and $l_{\text {num }}(t)$, computed from the numerical simulations (dotted lines) of the corresponding perturbed $\phi^{4}$ (upper panel) and sG equations (lower panel). Both systems are perturbed by a constant force $\epsilon=0.002$, and the initial parameters are $l(0)=l_{s}, \dot{l}(0)=0$, and $\dot{X}(0)=0$. Right: Evolution of the velocity of the kink center of the $\phi^{4}$ (upper) and sG (lower) kinks. The theoretical and numerical results for the velocity overlap, their difference being negligible at this scale.

is $\omega=1.2177$, very close to $\Omega_{\mathrm{i}}$. We can then conclude that what we see is the $\phi^{4}$ internal mode excitation entirely due to the initial distortion: recall that no oscillations were seen when the simulation began with the unperturbed kink. The situation is totally different in the sG case, for which DFT shows that $l_{\text {num }}(t)$ oscillates with a frequency $\omega=1.0073$, whereas the frequency of the CC prediction is $\omega_{\mathrm{CC}}=1.1312$. We thus see that the oscillation frequency is much closer to the lowest phonon mode $\omega_{1}=\sqrt{1+(2 \pi / 100)^{2}}=1.0019$ than to the Rice frequency $\Omega_{\mathrm{R}}=1.1026$. It thus becomes evident that the behavior of the $\mathrm{sG}$ kink is completely different from that of the $\phi^{4}$ kink, which is an indication that the internal mode of the latter is absent in the former. In addition, Figure 4 shows that when we increase the external force the oscillations of $l_{\text {num }}(t)$ are damped more rapidly than in the theoretical prediction for $l(t)$, for both $\phi^{4}$ and $\mathrm{sG}$ kinks. This occurs because those larger values of $\epsilon$ induce the appearance of radiation in the system, to which the kink loses its initial distortion energy more efficiently than if this energy would only be damped out by the force. As the $\mathrm{CC}$ method does not take radiation into account, it is obvious that its predictions cannot be accurate when there is radiation excited in the system.

As we have seen, our results suggest that whereas the $\phi^{4}$ kink possesses an internal mode that is excited by initial distortions of its shape, the sG kink does not have such a mode. In order to confirm this result, we turn to the case when there are no forces in the system but we start from a distorted kink $\left(l(0) \neq l_{s}\right.$ or / and $\left.i(0) \neq 0\right)$. In this case, shown in Figure 5, DFT shows that the CC prediction for $l(t)$ oscillates exactly with the Rice frequency, i.e., $\omega_{\mathrm{CC}}=\Omega_{\mathrm{R}}$ for both $\phi^{4}$ and $\mathrm{sG}$. The plot makes clear that the theoretical oscillations are very similar to the numerically simulated ones for the $\phi^{4}$ kink, where in this case $l_{\text {num }}(t)$ oscillates with $\omega=1.2272$, practically equal to the internal mode frequency $\Omega_{\mathrm{i}}$. The oscillations do not decay even for large times, as shown in the upper panel of Figure 6 . No signatures of other frequencies are found [23]. Again, the results are different for the sG kink: 

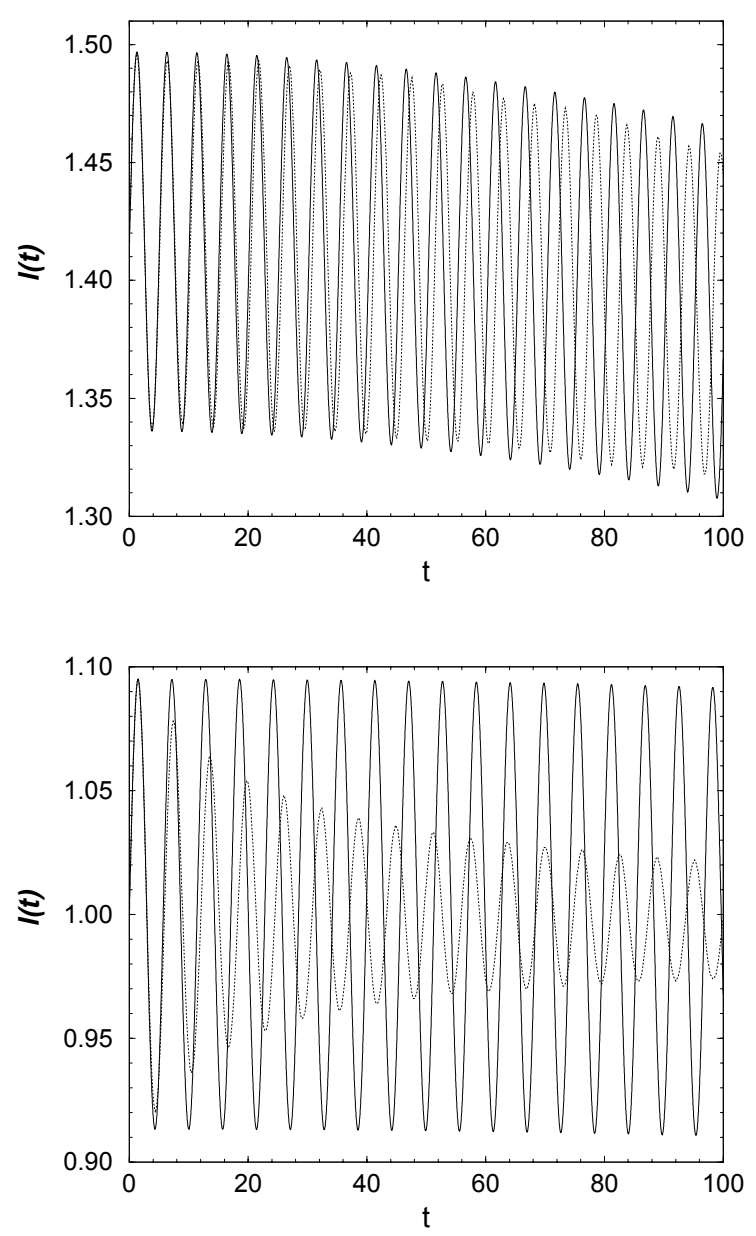

Fig. 2. Evolution of the kink width, $l(t)$, theoretically obtained from equations $(17,18)$ (solid lines), and $l_{\text {num }}(t)$, computed from the numerical simulations (dotted lines) of the corresponding perturbed $\phi^{4}$ (upper panel) and sG equations (lower panel). Both systems are perturbed by a force $\epsilon=0.001$, and the initial parameters are $l(0)=l_{s}, \dot{l}(0)=0.1$, and $\dot{X}(0)=0$.

In Figure 5 we see that $l_{\text {num }}(t)$ oscillates not only with a different frequency, $\omega=1.0058$, but also that the oscillations are rapidly damped. Note that the numerical $\omega$ is approximately equal to the lowest phonon frequency $\omega_{1}$. The evolution of $l_{\text {num }}(t)$ for larger times leads to the appearance of more frequencies (see Fig. 6): $\omega_{d}=0.9983$, $\bar{\omega}_{1}=1.0034, \bar{\omega}_{2}=1.0083, \bar{\omega}_{3}=1.0184, \bar{\omega}_{4}=1.0335$, $\bar{\omega}_{5}=1.0511, \bar{\omega}_{6}=1.0712$ and $\bar{\omega}_{7}=1.0963$. All the $\bar{\omega}_{i}$, except $\omega_{d}$, are related with the low-lying phonon modes: $\omega_{1}=1.0019, \omega_{2}=1.0079, \omega_{3}=1.0176, \omega_{4}=1.0311$, $\omega_{5}=1.0482, \omega_{6}=1.0687, \omega_{7}=1.0924$. As for $\omega_{d}$, it is is very close to the frequency of the internal mode induced by discreteness, analytically calculated in [9]:, $\omega_{i} \approx \sqrt{1-(4 / 2025)(\Delta x)^{4}}=0.999998$ for $\Delta x=0.05$. Therefore, we do not find any evidence of a quasimode or any similar long-lived mode and, as the $\phi^{4}$ results show, we would certainly have found it if it existed.

Our last results concern the effect of the dissipation in the system, predicted by the CC method. As in this case
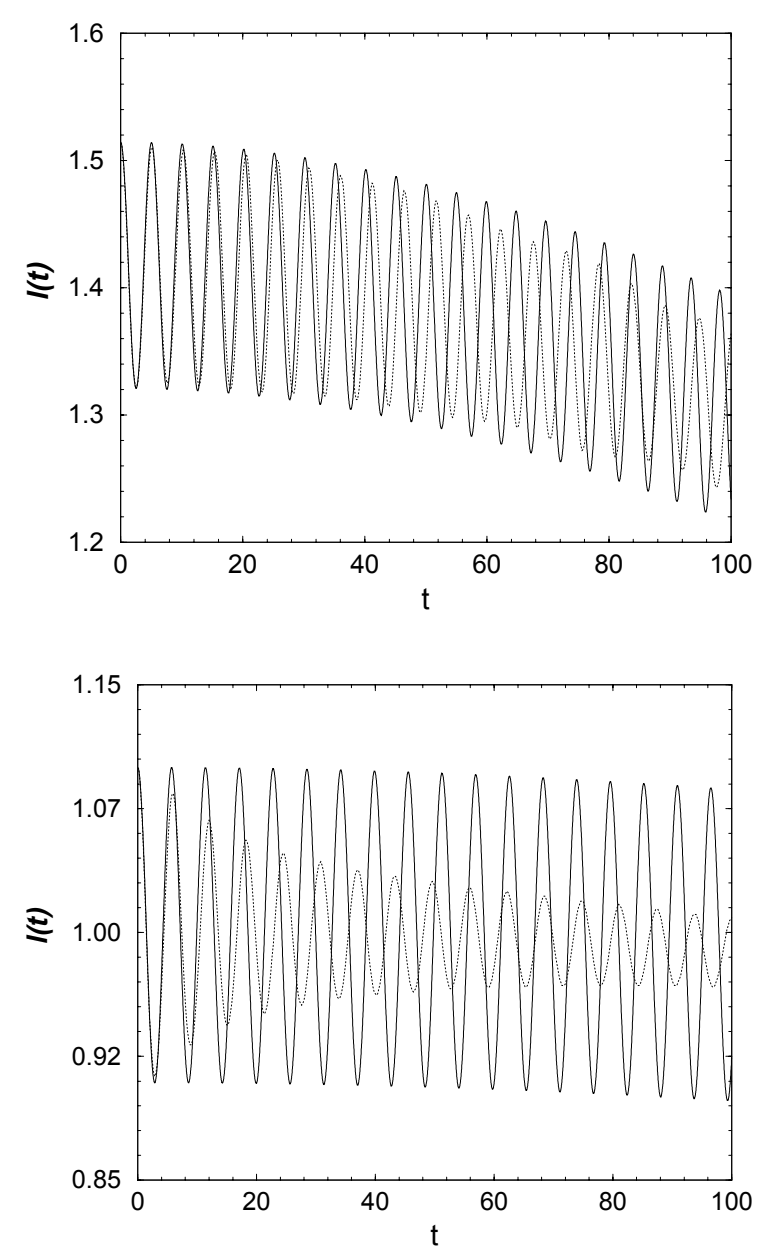

Fig. 3. Evolution of the kink width, $l(t)$, theoretically obtained from equations $(17,18)$ (solid lines), and $l_{\text {num }}(t)$, computed from the numerical simulations (dotted lines) of the corresponding perturbed $\phi^{4}$ (upper panel) and sG equations (lower panel). Both systems are perturbed by a force $\epsilon=0.001$, and with $l(0)=l_{s}+0.1, \dot{l}(0)=0$ and $\dot{X}(0)=0$.

we have not been able to solve the corresponding equations, we integrate numerically equation (5) with a 4-order Runge-Kutta method [24] with $\Delta t=0.01,0.001$; results are the same in both cases, so we are confident that we are correctly integrating the equation. The theoretical results in the figures are represented for $\Delta t=0.001$. In Figure 7 we see that the dissipation makes the amplitude of the oscillations of $l(t)$ rapidly decrease in time. For comparison, note that we have chosen the same values of $\epsilon, \dot{X}(0), l(0)$ and $i(0)$ as in Figure 4. We have also observed that if we increase $\beta$, the oscillations are damped even more rapidly. Turning now to the full equations, let us recall that if we introduce dissipation, $\beta=0.1$ in this case, the radiation almost disappears and therefore a better agreement with the CC predictions is to be expected. Figure 7 shows that this is indeed what occurs for the $\phi^{4}$ kink: we observe that $l_{\text {num }}(t)$ oscillates with a frequency $\omega=1.2177 \approx \Omega_{\mathrm{i}}$ while, simultaneously, the amplitude of these oscillations decreases due to the dissipation of the system. For the $\mathrm{sG}$ 

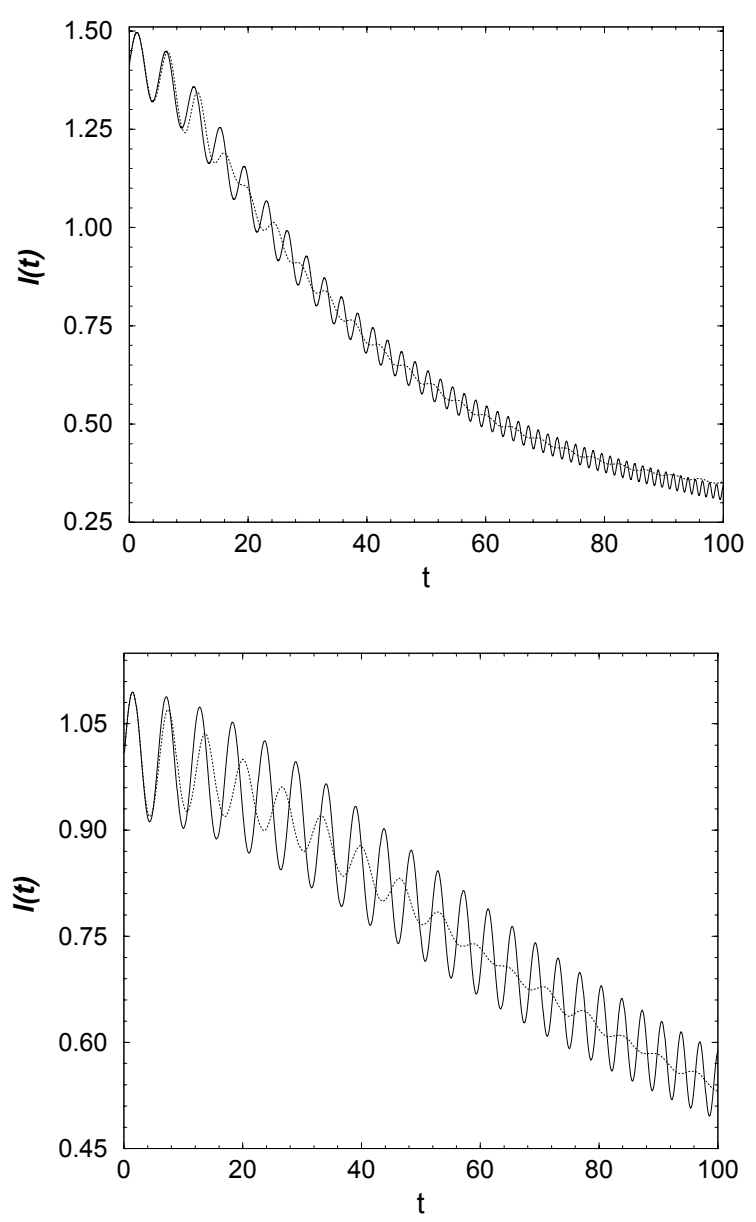

Fig. 4. Evolution of the kink width, $l(t)$, theoretically obtained from equations $(17,18)$ (solid lines), and $l_{\text {num }}(t)$, computed from the numerical simulations (dotted lines) of the corresponding perturbed $\phi^{4}$ (upper panel) and sG equations (lower panel). Both systems are perturbed by a force $\epsilon=0.02$, with $l(0)=l_{s}, \dot{l}(0)=0.1$, and $\dot{X}(0)=0$.

model (lower panel) the oscillations of $l_{\text {num }}(t)$ are smaller than the predicted ones by the $\mathrm{CC}$ approach, and once again if we observe that its frequency $\omega=1.0073$, closer to $\omega_{1}=1.0019$, so it is related neither with $\Omega_{\mathrm{R}}$ nor with a possible quasimode.

\section{Conclusions}

In this paper, we have studied analytically and numerically the behavior of $\phi^{4}$ and sG kinks subjected to constant external forces and damping. Analytically, we have developed a CC theory for the time evolution of the kink center and width, obtaining the same equations of motion as in reference [12]. For the dissipation-free case, we have found the exact solution of those equations for arbitrary initial conditions, thus generalizing the partial results in [12], this being our first novel result. Afterwards,
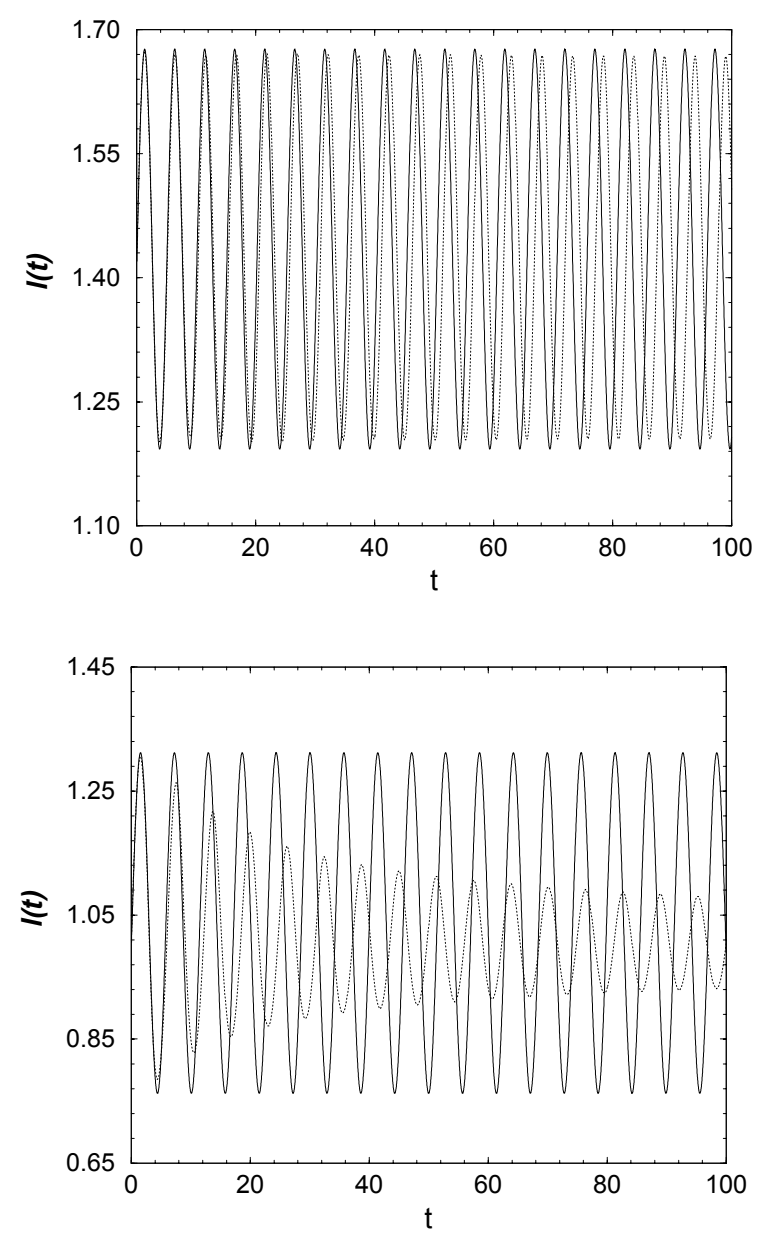

Fig. 5. Evolution of the kink width, $l(t)$, theoretically obtained from equations $(17,18)$ (solid lines), and $l_{\text {num }}(t)$, computed from the numerical simulations (dotted lines) of the corresponding perturbed $\phi^{4}$ (upper panel) and sG equations (lower panel). Both systems are unperturbed, $\epsilon=0$, with $l(0)=l_{s}$, $\dot{i}(0)=0.3$, and $\dot{X}(0)=0$.

we have verified the theoretical predictions so obtained by comparing them with numerical simulations, taking proper care of computing correctly the magnitudes of interest. Such comparison allowed us to establish some important conclusions which we summarize in what follows.

As we have seen, both the theoretical predictions and the numerical simulations show that constant external forces and dissipation, by themselves, are not able to excite any oscillation of the kink width. Furthermore, they are not even needed to excite those oscillations: In the absence of any other perturbation in the system, i.e., in kink propagation governed by the unperturbed $\mathrm{sG}$ or $\phi^{4}$ equation, an initial distortion of the kink width, either directly or through its derivative, is enough to excite width oscillations. Hence, we believe that the earlier work [12] on this subject, being formally correct, was somewhat misleading, as it suggested that the kink width oscillations were caused by the perturbations we are considering. It is now 

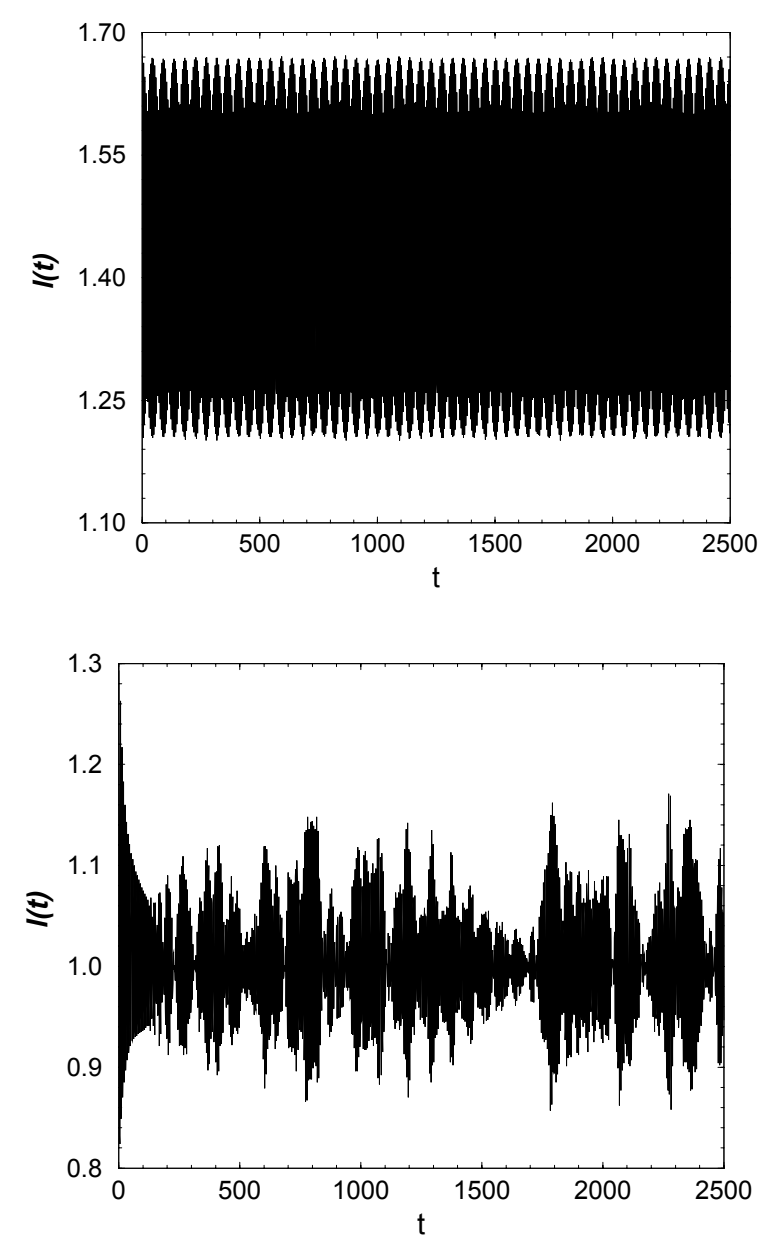

Fig. 6. Evolution of $l(t)$ for large times. Parameters are as in Figure 5.

clear that the effect of constant forces and damping is exactly the opposite: They damp away the width oscillations originated by the initial kink distortion.

For the $\phi^{4}$ kink, we have found that if they are let to evolve from an initially distorted shape, they do experience shape oscillations with a frequency which, in the absence of perturbations, is very close to the frequency of the internal mode. Perturbations modify slightly this frequency, but it remains close to that of the internal mode. We have verified that these oscillations are very long lived, and only after very long simulation times the combination of numerical errors and discreteness induced radiation acts on the width oscillations, making them decrease. In any case, we have established clearly that all the phenomenology discussed for the $\phi^{4}$ kink can be perfectly understood and described in terms of its intrinsic internal mode.

Finally, for the sG kink, the situation is completely different. The CC approach yields a very poor description of the numerically observed oscillations, which show
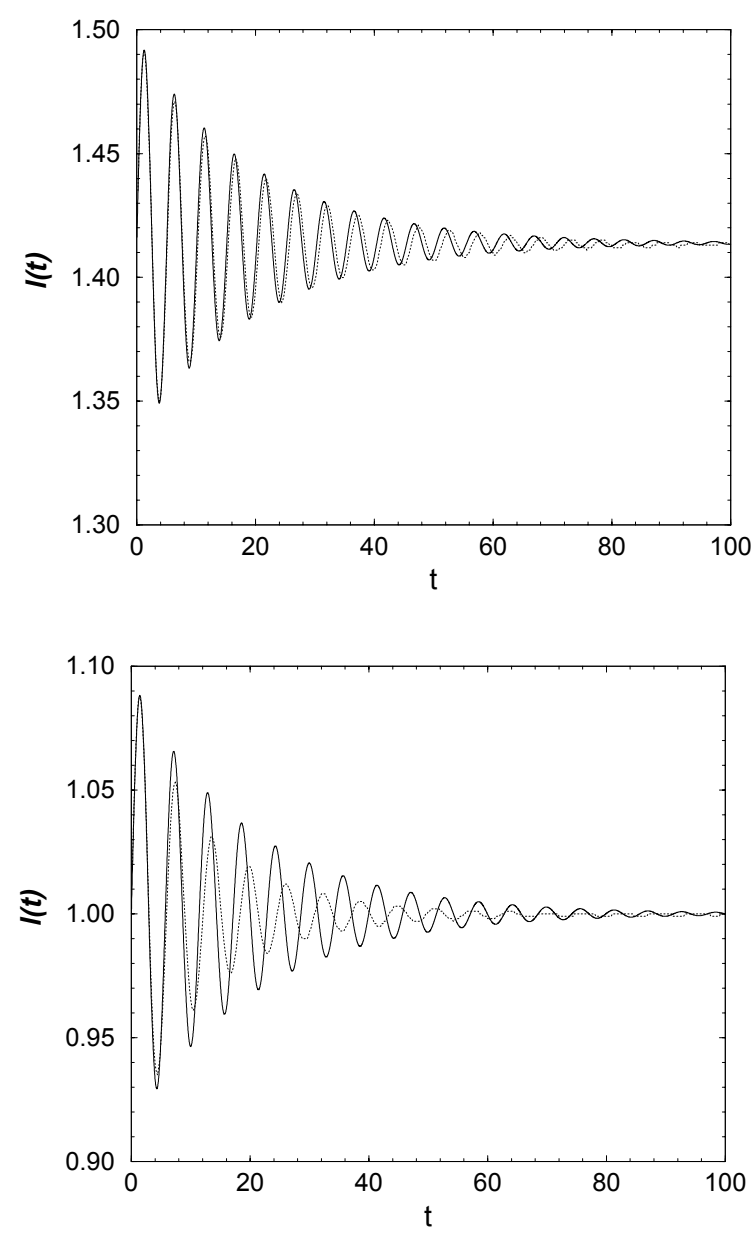

Fig. 7. Evolution of the kink width, $l(t)$, theoretically obtained from equations $(17,18)$ (solid lines), and $l_{\text {num }}(t)$, computed from the numerical simulations (dotted lines) of the corresponding perturbed $\phi^{4}$ (upper panel) and $\mathrm{sG}$ equations (lower panel). Both systems are perturbed with a constant force $\epsilon=0.02$ and damping $\beta=0.1$, with parameters $l(0)=l_{s}$, $\dot{l}(0)=0.1$, and $\dot{X}(0)=0$.

a very different frequency, are damped away much more rapidly, and in long runs end up involving several frequencies. These results confirm our earlier claim [13] that sG kinks do not possess any intrinsic internal mode (which was the hypothesis of the CC approach, as suggested before $[10,11])$. This conclusion stems from the comparison to the results of the $\phi^{4}$ kink: An initial distortion, with no special amplitude and no frequency, directly excited the internal mode, selecting only its frequency among the (in principle) many possible ways to use the additional energy of the distortion. However, in the sG case the same initial distortion was only able to excite radiation modes. Interestingly, we have also found evidence for the excitation of the internal mode induced by the discreteness of the simulation as calculated in [9]; this means that the procedure does indeed excite every available internal mode, and hence our claim that the sG kink does not have intrinsic internal modes, which, if present, would have been set in motion by the deformed initial conditions. This result fully 
agrees with the analysis presented in [13] for the case of ac drivings, and we believe that it settles definitively the question of the existence of quasimodes of $\mathrm{sG}$ kinks with a negative answer.

Work at GISC (Leganés) has been supported by DGESIC (Spain) grant PB96-0119 and by the grant No. BFM20000006. Travel between Bayreuth and Madrid was supported by "Acciones Integradas Hispano-Alemanas", a joint program of DAAD (Az. 314-AI) and DGESIC.

\section{References}

1. A.C. Scott, Nonlinear Science (Oxford University, Oxford, 1999).

2. Yu.S. Kivshar, B.A. Malomed, Rev. Mod. Phys. 61, 763 (1989), and references therein.

3. A. Sánchez, A.R. Bishop, SIAM Review 40, 579 (1998), and references therein.

4. M. Peyrard, D.K. Campbell, Physica D 9, 33 (1983); D.K. Campbell, J.F. Schonfeld, C.A. Wingate, ibid. 9, 1 (1983); D.K. Campbell, M. Peyrard, P. Sodano, ibid. 19, 165 (1986).

5. Yu.S. Kivshar, F. Zhang, L. Vázquez, Phys. Rev. Lett. 67, 1177 (1991); Phys. Rev. A 46, 5214 (1992).

6. Yu. Gaididei, T. Kamppeter, F.G. Mertens, A.R. Bishop, Phys. Rev. B 59, 7010 (1999).

7. N.R. Quintero, A. Sánchez, F.G. Mertens, Phys. Rev. Lett. 84, $871(2000)$
8. Yu.S. Kivshar, D.E. Pelinovsky, T. Cretegny, M. Peyrard, Phys. Rev. Lett. 80, 5032 (1998).

9. P.G. Kevrekidis, C.K.R.T. Jones, Phys. Rev. E 61, 3114 (2000).

10. M.J. Rice, Phys. Rev. B 28, 3587 (1983).

11. R. Boesch, C.R. Willis, Phys. Rev. B 42, 2290 (1990).

12. E. Majerníková, Yu.B. Gaididei, O.M. Braun, Phys. Rev. E 52, 1241 (1995).

13. N.R. Quintero, A. Sánchez, F.G. Mertens, Phys. Rev. E 62 Rapid Comm., R60 (2000).

14. F.G. Mertens, H.J. Schnitzer, A.R. Bishop, Phys. Rev. B 56, 2510 (1997).

15. N.R. Quintero, A. Sánchez, F.G. Mertens, Phys. Rev. E 62, 5695 (2000).

16. J.L. Reid, J.R. Ray, Z. Angew. Math. Mech. 64, 365 (1984).

17. E. Pinney, Proc. Amer. Math. Soc. 1, 681, (1950).

18. E.T. Whittaker, G.N. Watson, A course of Modern Analysis (Cambridge University Press, 1984).

19. A.W. Babister, Transcendental Functions Satisfying Nonhomogeneous Linear Differential Equations (MacMillan, 1967).

20. W.A. Strauss, L. Vázquez, J. Comput. Phys. 28, 271 (1978).

21. S. Jiménez, L. Vázquez, Appl. Math. Comput. 35, 61 (1990).

22. N.R. Quintero, A. Sánchez, F.G. Mertens, Phy. Rev. E, 60, 222 (1999).

23. Only for larger times, greather than $t \approx 10000$, we have seen that the amplitude of those oscillations decreases, while the center of the kink begins to move, a phenomenon that it is probably due to the accumulation of numerical errors and the corresponding excitation of linear radiation modes, or to higher order effects coupling the internal and the translation mode through discreteness.

24. W.H. Press, S.A. Teukolsky, W.T. Vetterling, B.P. Flannery, Numerical Recipes in Fortran, 2nd edn. (Cambridge University Press, Cambridge, 1992). 